This is a post-peer-review, pre-copyedit version of an article published in Molecular Plant. The final authenticated version is available online at: https://doi.org/10.1016/i.molp.2021.02.008

\title{
The mechanism of auxin transport in lateral root spacing
}

Authors: Jian Chen ${ }^{1,2, *}$, Hugues De Gernier ${ }^{1,2,{ }^{*}}$, Alaeddine Safi ${ }^{1,2}$, Tom Beeckman ${ }^{1,2, *}$ and Steffen Vanneste $\mathrm{e}^{1,3,4, \#}$

${ }^{1}$ Department of Plant Biotechnology and Bioinformatics, Ghent University, 9052 Ghent, Belgium

${ }^{2}$ Center for Plant Systems Biology, VIB, 9052 Ghent, Belgium

${ }^{3}$ Lab of Plant Growth Analysis, Ghent University Global Campus, Incheon 21985, Republic of Korea

${ }^{4}$ Department of Plants and Crops, Ghent University, 9000 Ghent, Belgium

*These authors contributed equally

\# for correspondence: tobee@psb.vib-ugent.be; steffen.vanneste@ugent.be

Transport of the plant hormone auxin (Indole-3-acetic acid; IAA) is crucial to ensure proper growth patterns. In particular, the regular spacing of lateral roots (LRs) is governed by intricate auxin movements and an internal oscillator, called the 'root clock' (Xuan et al., 2020). Multiple auxin influx and efflux carriers have been characterized in the broad context of root development. However, one of the remaining challenges is to puzzle out the auxin transport machinery that regulates the root clock and LR spacing.

One of the first indications of the central role for auxin transport in LR spacing came from the observation that the auxin transport inhibitor 1-NapthylPhtalamic Acid (NPA) inhibits LR initiation in Arabidopsis (Casimiro et al., 2001). While this inhibitor indiscriminately inhibits 
shootward and rootward auxin transport, it was at that time already proposed that rootward auxin transport is involved LR spacing. Subsequent research led to the concept of a root clock that instructs the spatio-temporal LR distribution (Xuan et al., 2020). The output of such oscillator is the periodic formation of auxin response maxima in Arabidopsis shootward of the apical meristem, followed by LR organogenesis, when a critical amplitude is reached (Fig. 1). Interestingly, the amplitude and periodicity of the oscillatory auxin response depend on auxin derived from the lateral root cap (LRC) via a NPA-sensitive mechanism (Xuan et al., 2020). This therefore suggests that the root clock is controlled by auxin transport. Additionally, mutant analyses and tissue-specific complementation studies show that the oscillatory auxin response amplitude most likely reflects auxin signaling in the stele, rather than in the outer tissues (Xuan et al., 2020). The spatial separation between the auxin source (LRC) and site of auxin perception (stele), thus suggests the additional involvement of radial inward auxin transport across the epidermis, cortex and endodermis in tuning the root clock (Fig. 1)

Genetic and molecular analyses have allowed developing in silico models that reproduce realistic auxin accumulation patterns in the root, suggesting that they capture the main auxin transport routes in the root. This thus allows interrogating the individual contributions of different auxin influx and efflux transporters in the model to the root clock. Consistently with the model, the auxin uptake activity of the IAA $/ \mathrm{H}^{+}$symporter AUX1 in the lateral root cap (LRC) could be experimentally validated as important for LR spacing (Xuan et al., 2020). In contrast, none of the mutants in ABCB- and PIN-type auxin transporters with predicted key roles in auxin efflux in the model display reduced LR formation. This illustrates an important gap in our understanding about how auxin transport dictates LR positioning via the root clock.

\section{Closing in on the auxin efflux component via D6PK kinases and auxin transport inhibitors}

To date, the strongest experimental evidence for the involvement of auxin efflux transporters in regulating the root clock derives from the chemical auxin transport inhibitors NPA and BUM (2-[4-(diethylamino)-2-hydroxybenzoyl]benzoic acid) (Casimiro et al., 2001; Xuan et al., 2020). Therefore, these chemicals can help identifying the auxin transporter mechanism that control the root clock. 
Both NPA and BUM likely interfere with ABCB-mediated auxin transport via disruption of the association of $A B C B$ s with the regulatory immunophilin-like FKBP42, TWISTED DWARF1 (Bailly et al., 2008). Recently, NPA was also proposed to inhibit PIN-mediated auxin transport via direct interaction (Abas et al., 2021) and altered PIN dimerization (Abas et al., 2021; Teale et al., 2021). However, no mutants in PINs or ABCBs phenocopy the NPA root clock phenotype.

Higher order mutants of members of a group of AGC kinases (D6PK) regulating PINmediated auxin transport lack LRs (Barbosa et al., 2018). The central role of D6PK activity in LR development is corroborated by the characterization of D6PK-regulatory PDK1/2 kinases, whose double mutant is also defective in LR development (Tan et al., 2020). Notably, the expression patterns of the synthetic auxin response reporter DR5::GUS in $d 6 p k$ and $p d k 1 / 2$ mutant roots correspond to those seen after NPA treatment (Xiao and Offringa, 2020). Moreover, $d 6 p k$ mutant root elongation is hypersensitive to NPA, suggesting that D6PK also affects the root clock. An indirect link between D6PK activity and root clock regulation can be inferred from the rapid plasma membrane dissociation and inactivation of D6PKs in response to the fungal toxin Brefeldin A (Barbosa et al., 2018). This response depends on BFA's ability to inhibit the ADP-ribosylation Factor-Guanine Exchange Factor (ARF-GEF) GNOM (Barbosa et al., 2018), which has recently been shown to be essential for the root clock function (Wachsman et al., 2020).

D6PKs can activate PIN-mediated auxin transport via direct phosphorylation (Barbosa et al., 2018). Interestingly, phosphomimetic substitutions that overlap with a D6PKphosphorylation site seem to modify PIN1's capacity to dimerize (Teale et al., 2021). This suggest that NPA and D6PK antagonize each other at the level of PIN-mediated auxin transport, highlighting a potential involvement of PINs in the root clock (Fig. 1). However, the function of PINs in the root clock could not yet be determined due to functional redundancy and pleiotropic developmental defects in higher order pin mutants. Alternatively, it is possible that other joint targets control the root clock (Fig. 1).

\section{Evolutionary perspective of auxin-transport independent LR spacing mechanisms}


Lateral root initiation is not universally sensitive to NPA. In late diverged ferns, such as Ceratopteris richardii, NPA has no impact on LR initiation, nor on its morphogenesis (llina et al., 2018). What is more, the lack of NPA binding in PINs of green algae (Klebsormidium flaccidum) (Abas et al., 2021), and shootward auxin transport in Ceratopteris richardii roots being largely NPA-insensitive (Zhang et al., 2019), is consistent with an independent evolutionary origin of lateral root branching in ferns (Hetherington et al., 2020).

Despite the putative single evolutionary origin of lateral root branching in the seed plant lineage (Hetherington et al., 2020), not all seed plants display NPA-sensitive LR initiation. An example of this is pumpkin (Cucurbita pepo), in which NPA does not abolish LR initiation, but impairs LR morphogenesis (Ilina et al., 2018), suggesting auxin transport dependence of the latter, but not the former. Interestingly, LR initiation in Cucurbita pepo occurs in close proximity of the root stem cell region, while LR initiation in Arabidopsis, and the bulk of other seed plants occurs distal to the meristem, explaining a differential dependence for auxin transport in both types of LR spacing mechanisms. Remarkably, the most primitive root branching mechanism that could be detected in the extinct lignophytes (seed plants and their seedless ancestors that produced wood lineage) is root bifurcation, a process that is occurring at the very tip of the meristem (stem cell region). This is consistent with the proposal that LR formation as seen in Cucurbita pepo, corresponds to an evolutionary intermediate between bifurcation and Arabidopsis-type LR formation (Ilina et al., 2018). During this evolution, NPA-sensitive auxin transport seems to be recruited to maintain communication between the meristem and the more distal site of LR initiation.

\section{Remaining questions and future research directions}

Even after 20 years of research, the auxin transport mechanism that controls LR spacing remains to be elucidated. Recent findings on NPA targets and PDK-D6PK activity strongly point to the involvement of PINs. However, their involvement in the root clock has been difficult to assess. We anticipate that the advent of tissue-specific and inducible genome-editing approaches (Wang et al., 2020) will allow overcoming pleiotropic developmental defects in higher order pin mutants, and finally answer this open question in developmental biology. 


\section{Acknowledgements:}

This work is funded by the Chinese Science Council (CSC) (J.C; 201606690035), Special Research Fund Ghent University - Postdoctoral fellowship at Ghent University (to H.D.G: BOF20/PDO/011) and FWO (G024118N).

\section{References}

Abas, L., Kolb, M., Stadlmann, J., Janacek, P. D., Lukic, K., Schwechheimer, C., Sazanov, L. A., Mach, L., Friml, J., and Hammes, U. Z. (2021). Naphtylphtalamic acid associates with and inhibits PIN auxin transporters. Proc Nat1 Acad Sci U S A 118:e2020857118.

Bailly, A., Sovero, V., Vincenzetti, V., Santelia, D., Bartnik, D., Koenig, B. W., Mancuso, S., Martinoia, E., and Geisler, M. (2008). Modulation of $\mathrm{P}-\mathrm{glycoproteins}$ by auxin transport inhibitors is mediated by interaction with immunophilins. J Biol Chem $283: 21817-21826$.

Barbosa, I. C. R., Hammes, U. Z., and Schwechheimer, C. (2018). Activation and Polarity Control of PIN-FORMED Auxin Transporters by Phosphorylation. Trends Plant Sci 23:523-538.

Casimiro, I., Marchant, A., Bhalerao, R. P., Beeckman, T., Dhooge, S., Swarup, R., Graham, N., Inze, D., Sandberg, G., Casero, P. J. , et al. (2001). Auxin transport promotes Arabidopsis lateral root initiation. Plant Cel1 13:843-852.

Hetherington, A. J. , Berry, C. M. , and Dolan, L. (2020). Multiple origins of dichotomous and lateral branching during root evolution. Nat Plants 6:454-459.

Ilina, E. L., Kiryushkin, A. S., Semenova, V. A., Demchenko, N. P., Pawlowski, K., and Demchenko, K. N. (2018). Lateral root initiation and formation within the parental root meristem of Cucurbita pepo: is auxin a key player? Ann Bot 122:873-888.

Tan, S., Zhang, X., Kong, W., Yang, X. L., Molnar, G. , Vondrakova, Z. , Filepova, R., Petrasek, J., Friml, J., and Xue, H. W. (2020). The lipid code-dependent phosphoswitch PDK1-D6PK activates PINmediated auxin efflux in Arabidopsis. Nat Plants 6:556-569.

Teale, W. D., Pasternak, T., Dal Bosco, C., Dovzhenko, A., Kratzat, K. , Bildl, W., Schworer, M., Falk, T., Ruperti, B., J, V.S., et al. (2021). Flavonol-mediated stabilization of PIN efflux complexes regulates polar auxin transport. EMB0 J 40:e104416.

Wachsman, G., Zhang, J., Moreno-Risueno, M. A., Anderson, C. T., and Benfey, P. N. (2020). Cell wall remodeling and vesicle trafficking mediate the root clock in Arabidopsis. Science 370:819-823. 
Wang, X., Ye, L., Lyu, M., Ursache, R., Loytynoja, A., and Mahonen, A. P. (2020). An inducible genome editing system for plants. Nat Plants 6:766-772.

Xiao, Y., and Offringa, R. (2020). PDK1 regulates auxin transport and Arabidopsis vascular development through AGC1 kinase PAX. Nat Plants 6:544-555.

Xuan, W., De Gernier, H., and Beeckman, T. (2020). The dynamic nature and regulation of the root clock. Development 147.

Zhang, Y., Xiao, G., Wang, X., Zhang, X., and Friml, J. (2019). Evolution of fast root gravitropism in seed plants. Nat Commun $10: 3480$.

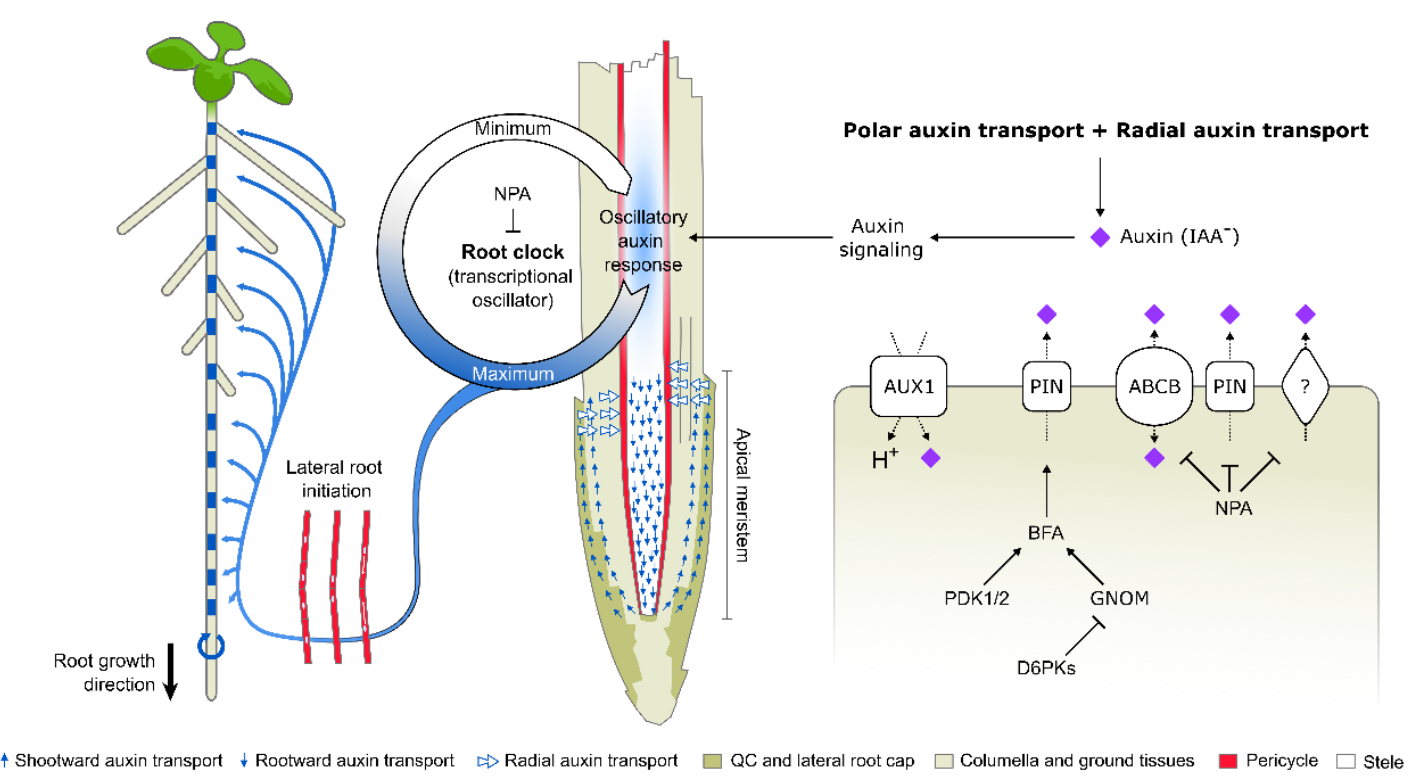

Figure 1: Auxin transport for LR spacing in Arabidopsis. The spacing of LRs along the axis of the root is instructed by the root clock, that is easily visualized by an oscillatory auxin response. At auxin response maxima, a new LR is initiated. The root clock depends on NPA sensitive auxin transporters and the AUX1 auxin uptake carrier. NPA inhibits at least the activities of ABCB- and PIN-type auxin transporters. PIN1 is regulated by D6PK kinases that are in turn regulated by PDK1/2 and GNOM, a BFA-sensitive ARF-GEF. 